\title{
Dreamtime and awakenings: facing realities of remote area Aboriginal health
}

\author{
Russell L Gruen and Theresa FM Yee \\ It takes humility to walk along the path towards better Aboriginal health
}

M arni and her two cousins crowded together on our couch watching the Saturday footy. Like many of the Aboriginal children who regularly visited us, they laughed and chatted and found fun in almost everything. We were all still damp after a cooling swim at the nearby waterhole, and were about to tuck into a freshly baked cake from our oven. It seemed there could be nothing better.

But 8-year-old Marni was unusually breathless. In fact, she was wheezing and gasping for air. With a stethoscope we could hear the crackles of pulmonary oedema. A few months earlier she had spent several weeks in hospital, and there had been talk that she might need valve replacement surgery. She narrowly avoided it then. Now her cardiac failure had worsened again.

Marni has rheumatic heart disease - a disease of poverty and overcrowding. We had never seen a case before we went to work in Yambarr. However, in this small community of about 900 people, at least 25 have rheumatic heart disease.

As doctors, we had sought the challenges of working in a remote Aboriginal community. Admittedly, we also found some romantic appeal in the mythical and timeless aspects of Aboriginal culture, which are said to embrace the earth, its creatures and the spirit world in what is often referred to as The Dreamtime. Inevitably, however, we found real people living lives of hardship. More than finding The Dreamtime, we were awakened to some stark realities about health in remote communities.

\section{Yambarr Aboriginal community}

Yambarr is hundreds of kilometres from the nearest city. From the air, the collection of tin roofs and shady trees is hardly noticeable in the rugged landscape traversed by vein-like rivers quivering in the 40 degree heat. Closer up, a permanent waterhole can be seen, flanked by scattered houses, a school, a store and a police station, and the ramshackle old clinic.

The clinic's exterior is adorned with traditional paintings of animals and birds, once radiant but now faded and peeling, weathered by years of monsoonal storms and grassfires. The doors and windows are barred, and ageing plywood inserts take the place of occasional missing panes. Standing outside, hearing only the rattle of straining air conditioners, it seems a long way from modern specialised medical centres.

The names and other details of people and places have been altered to protect and respect the privacy of individuals and communities.

Menzies School of Health Research, Institute of Advanced Studies, Charles Darwin University, Casuarina, NT.

Russell L Gruen, MB BS, PhD, FRACS, Associate Professor;

Theresa FM Yee, MB BS, FRACGP, General Practitioner.

Reprints will not be available from the authors. Correspondence: Associate Professor Russell L Gruen, Menzies School of Health

Research, PO Box 41096, Casuarina, NT 0811.

russell.gruen@menzies.edu.au
Inside, however, activity thrives in the relative coolness. Patients, families, and staff mill among the five consulting rooms, the drug dispensary and the emergency room. Lining the corridor are posters of Aboriginal athletes and footballers bringing health messages. This clinic has three nurses and six Aboriginal health workers - men and women who work as clinicians, interpreters and cultural aids for transient non-Aboriginals like us.

Non-Aboriginal people have been in the area for a little over 100 years, a tick of the clock compared with the more than 40000 -year history of Aboriginal people. Nevertheless, white traders, missionaries and developers have left no corner untouched. Indigenous communities today are melting pots of traditional and modern ways. Coca-Cola and ice-cream go well with a meal of fresh kangaroo. Faded jeans, Nike shoes and stereos are just part of the scene at tribal dances and cultural ceremonies. Children like Marni seem to embrace both their traditional and modern worlds with an ease and simplicity that only children know.

But the postcard pictures of painted faces, spears and boomerangs conceal some unspeakable suffering. Tucked away in the remote outback, communities like Yambarr are Australia's equivalent of urban slums, where poverty, illness, illiteracy and unemployment coalesce in vicious circles. Nationally, Aboriginal people die on average 20 years earlier than other Australians, half before the age of 50 years. Rates of chronic disease and substance abuse are increasing. On nearly every social, economic and health indicator Aboriginal people are much worse off.

\section{The human faces of disadvantage}

Living in Yambarr we saw these statistics in the faces of our friends, neighbours and even the clinic staff themselves. Within months of our arrival, two important Aboriginal men in the community had myocardial infarctions. One was a health worker and the other a community councillor. Both were still in their 20s. Never had we seen AMIs at such a young age. The first, Elijah, died only minutes after he had been laughing with us in the tearoom during a busy morning clinic. Then he was on the floor, his lifeless eyes staring at us, surrounded by monitors, tubes and wailing patients. We had no defibrillator, and he died amidst our helplessness.

Only weeks later Bill collapsed during a football match and his team-mates carried him in, his black face pale with fear. Bill had been our chaperone when we arrived in the community, ensuring that we were welcome and safe, involving us in community activities and including us into his family. He survived his heart attack, receiving thrombolytic treatment as he lay on a trolley at Yambarr's desert airstrip, shaded from the sun by the wing of a twin-propeller air ambulance.

Such inexplicable events occurred with tragic frequency. The statistics dissolved into the faces of people we knew — at work, in the street, down at the shop, hanging around the playground. 


\section{CLINICAL CONCERNS - PERSONAL PERSPECTIVE}

From a medical perspective, three words seemed to describe our patients' lives (in so far as words could capture them) - "hardship", "sufferance" and "invisibility".

Hardship refers to the struggle of daily living. In Yambarr 900 people live in fewer than 70 dwellings, lined up in neat rows and differing only in the colour of their faded exteriors. Bill's mother, Marjorie, rents a three-bedroom, orange-painted house covered with graffiti. Its concrete floors are cracked, its window screens are broken, and the plumbing occasionally blocks. Bill lives there with his family, and so do his brother and sister and their families, usually more than a dozen people. The children play in the small yard - mud in the wet season and dust in the dry - among freeroaming, unhealthy looking dogs that pick through scraps. Both Bill and his brother have been wait-listed to rent another home for over 10 years, but a new house has not become available.

Despite the popularity of hunting and fishing, families have become accustomed to shopping for food at the Yambarr store. Due to the heat and distance from markets, the supply of fresh fruit and vegetables is limited, and what is available is very expensive. Most families buy tinned produce, which may be high in fat and sugar. Most available hot food is greasy and fried. When Bill was told in hospital that he had impaired glucose tolerance, like at least 100 of the adults in Yambarr, he knew a suitable diet would be virtually impossible.

Alcohol is also part of the hardship, especially for children who grow up with the impression that drinking, often excessively, is a normal part of adult life. Youth programs have been short-lived, and in the end the only established gathering place for entertainment for anyone, including kids, is the licensed club.

For many Yambarr people there is simply no escape from the hardship of daily existence and few opportunities to improve their situation. Housing management, the running of the store and the activities of the club depend, to a greater or lesser degree, on externally provided non-Aboriginal contractors and administrators. Poor literacy is one of the biggest barriers to local Aboriginal people assuming real responsibility in imposed systems that are complex and unfamiliar. Bill and Elijah were quite exceptional in completing vocational training. A recent independent inquiry into Aboriginal education reported that only $4 \%$ of Aboriginal students in remote communities reach national reading benchmarks at fifthgrade level, compared with $80 \%$ of non-Aboriginal students. It also revealed that many leave school with English literacy and numeracy levels equivalent to 6 or 7 year olds and are, therefore, virtually unemployable. The fact that many are fluent in at least three or four other Aboriginal languages helps little when hunting for a mainstream job.

We wondered about this as Marni (despite her breathlessness) and her cousins laughed, smiled and chatted away on the couch. They variously attended school, sometimes every day, sometimes less frequently. Their parents all had an English-speaking education, but most were now unemployed, because few jobs existed in Yambarr, and the big city was a hostile, unfamiliar white-person's place away from family and friends. It's easy to imagine that they had seen little value in their own education, and hence were often indifferent about their children's school attendance.

Furthermore, it is well known that good nutrition and good hearing are essential for learning. Yet, in remote areas, 20\% of Aboriginal children younger than 5 years are underweight, and almost four out of five children have hearing disabilities. In some communities the prevalence of chronic otitis media has been recorded as 50\%, more than 10 times that which the World Health Organization regards as a significant public health problem.

Sufferance describes a resignation to illness that we found both perplexing and disturbing. We were amazed that families could quietly tolerate such sickness. Perhaps, in the context of so much disease, people grow up expecting illness as part of life. Perhaps they are unaware of available treatment. Many parents seemed unworried when pus oozed from their children's ears. Even Bill's cheerful 7-year-old daughter, Stephanie, had recurrent ear infections and perforated ear drums. Although Bill's mother was a health worker, both of them seemed to be as numbed by the sheer prevalence of illness as nearly everyone else in Yambarr. Over the years they had developed no better expectations.

Invisibility refers first to the level of undiagnosed and untreated illness in the community. Many chronic diseases, for example, do not become apparent to local health care providers until acute complications present. In Bill's case, it was only after his heart attack that he was found to have rheumatic heart disease. Rheumatic heart disease provides a good illustration of the invisibility of Aboriginal peoples' health problems to metropolitan specialist centres - despite our clinical experience, there remains a perception among some that rheumatic fever has been eradicated from Australia.

Invisibility also refers to our impression that, in many ways, progress has passed by communities like Yambarr, and that many well-meaning health professionals have little understanding of Aboriginal people's difficulties obtaining health care. Even when disease is recognised, barriers to accessing mainstream health services arise because of cultural differences, language and communication difficulties, remoteness and poverty. Forty per cent of Aboriginal communities are over 250 kilometres from the nearest hospital, most without regular public transport. From Yambarr, a simple 15-minute hospital appointment requires a day of travelling in each direction and two nights' accommodation at crowded urban hostels. A recent survey at one hospital's outpatient clinic showed that nearly two-fifths of people from remote communities did not keep their appointments. Non-attendees' appointments are usually rescheduled weeks later, and their travel subsidies are often forfeited.

The reasons for such non-attendance are rarely straightforward. We realised this after we sought a specialist ENT opinion for Stephanie. She and two other children waited 10 weeks for an initial appointment. Stephanie's grandmother accompanied them in the back of a Toyota Troopcarrier to meet the public bus about 200 kilometres away. This bus service provides both public transport and occasional tourism. The non-Aboriginal driver often gives an en-route commentary about Aboriginal culture, much to the amusement of any Aboriginal passengers. This time, however, we found the old lady and the three children still at the bus stop long after the bus had departed. Embarrassed and ashamed, they asked us for a ride home. They explained that the driver had refused to let them board because they had no shoes, despite the fact that Aboriginal people in Yambarr rarely wear them. The driver had promised that he would wait while they purchased some in a nearby store. When they returned with the footwear only a few minutes later, the bus was gone. On the way home we lamented the effort that went into organising their appointments, the disruption to their families and, above all, the fact that the children would have to wait another 2 months to see a specialist. 


\section{CLINICAL CONCERNS - PERSONAL PERSPECTIVE}

So, on top of the long-lasting health effects of poor nutrition, overcrowding, alcohol and unemployment, Aboriginal people struggle to obtain the full benefits of modern health care. Even at the Yambarr clinic, their health seemed to take one step forward while they were there and two steps back as soon as they left. Much of the clinic's work was "band-aid medicine" - a result of both insufficient resources and overwhelming social forces. As health professionals, we were humbled by our patients' hardship, sufferance and invisibility.

\section{A sense of humility}

Humility is not often discussed in the health professions. Everyone seems much more interested in modern medical triumphs. Humility instead refers to an awareness of one's limits. In theology, it refers to a sense of "smallness" in relation to God's greatness, encouraging receptiveness and solidarity with all people, especially the poor, "lowly" and "sinners". To us, in Yambarr, humility had secular relevance.

A sense of smallness was evident in many ways, including our limited ability to improve the health of Yambarr's people. It's not that our medical knowledge didn't provide answers to many health problems. It usually did, one person at a time. (Marni, for example, eventually had a successful valve replacement in a hospital thousands of kilometres away.) It's more that our training provided no simple answers to preventing the enormous burden of illness that existed in Yambarr or to overcoming the difficulties of getting modern health care to the people who need it most. Furthermore, problems of housing quality, alcohol-related harm and food quality often seemed insurmountable to the overstretched clinic staff. It would take a community-wide effort to improve these things.

We felt solidarity with our patients and with the community. Getting to know the people as friends and neighbours, we realised that we shared common human values and goals — things such as health, love and happiness, the centrality of family and the importance of community, and the need to imagine a bright future. With them, we experienced the pain and frustration of their medical conditions and their difficulties getting treatment. We reflected their sadness about intractable problems that made the future seem so uncertain.

Eventually, we found ourselves doing things that weren't part of ordinary medical practice, such as participating in community projects and lobbying for various hospital and community improvements. For example, the clinic staff joined with other community organisations to address scabies, a skin infection that is a risk factor for rheumatic heart disease. After developing screening, surveillance and treatment programs, and producing locally an educational video, the prevalence of scabies infections in children under the age of three fell from 33\% to 8\%.

At other times, after Elijah's death, we were advocates, lobbying for cardiac defibrillators in communities. After all, they are available in shopping centres, sporting venues and ambulances in many parts of the world. And the central health services agreed. By the time we left, many communities in the area had them. But solutions to many problems are not so simple. By knowing the difficulties our patients faced, however, we felt obliged to bring these to the attention of others who may be more able than we are to bring about lasting improvements in Aboriginal health and community life.

Doctors and other health professionals can do a lot for people in places like Yambarr, but not all of it uses tools from the traditional black bag. Much of it is about advocating for change, highlighting problems, and refusing to be passive about hardship, sufferance and invisibility. We learned that it takes humility to walk along the path towards better Aboriginal health. And most humbling of all is that, along that path, we have changed more than our patients.

(Received 20 Sep 2004, accepted 14 Dec 2004) 